\title{
Extended X-ray emission in PKS 1718-649
}

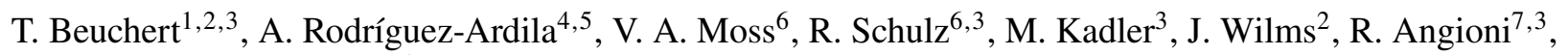 \\ J. R. Callingham ${ }^{6}$, C. Gräfe ${ }^{2}$, F. Krauß ${ }^{8}$, A. Kreikenbohm ${ }^{3}$, M. Langejahn ${ }^{3}$, K. Leiter ${ }^{3}$, \\ F. M. Maccagni ${ }^{9}$, C. Müller ${ }^{10,7}$, R. Ojha ${ }^{11}$, E. Ros ${ }^{7,12,13}$, and S. J. Tingay ${ }^{14}$

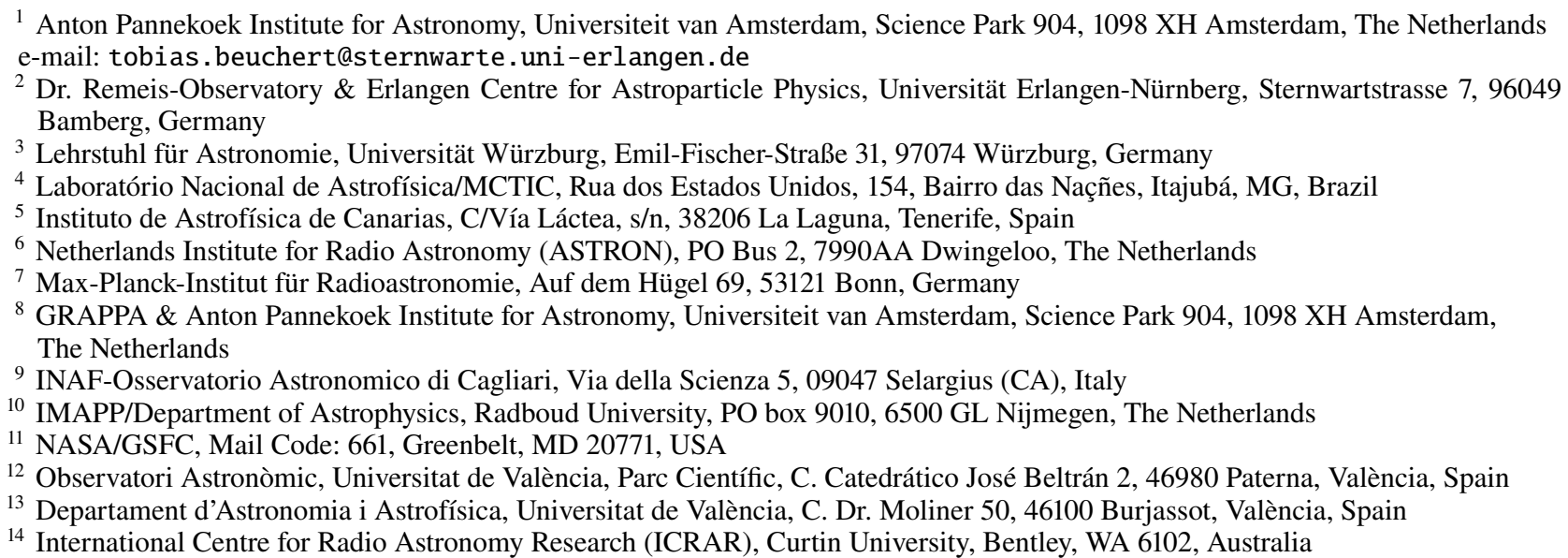

Received 20 March 2018 / Accepted 8 April 2018

\begin{abstract}
PKS 1718-649 is one of the closest and most comprehensively studied candidates of a young active galactic nucleus (AGN) that is still embedded in its optical host galaxy. The compact radio structure, with a maximal extent of a few parsecs, makes it a member of the group of compact symmetric objects (CSO). Its environment imposes a turnover of the radio synchrotron spectrum towards lower frequencies, also classifying PKS 1718-649 as gigahertz-peaked radio spectrum (GPS) source. Its close proximity has allowed the first detection of extended X-ray emission in a GPS/CSO source with Chandra that is for the most part unrelated to nuclear feedback. However, not much is known about the nature of this emission. By co-adding all archival Chandra data and complementing these datasets with the large effective area of XMM-Newton, we are able to study the detailed physics of the environment of PKS $1718-649$. Not only can we confirm that the bulk of the §kiloparsec-scale environment emits in the soft X-rays, but we also identify the emitting gas to form a hot, collisionally ionized medium. While the feedback of the central AGN still seems to be constrained to the inner few parsecs, we argue that supernovae are capable of producing the observed large-scale X-ray emission at a rate inferred from its estimated star formation rate.
\end{abstract}

Key words. galaxies: active - galaxies: nuclei - galaxies: individual: PKS 1718-649 - galaxies: ISM - galaxies: star formation X-rays: galaxies

\section{Introduction}

With a turnover at around $4 \mathrm{GHz}$, PKS $1718-649$ is a prominent representative of the class of gigahertz-peaked radio spectrum (GPS) sources (Tingay et al. 1997; Jauncey et al. 1998; Tingay \& de Kool 2003). It is one of the closest sources of its kind, with only NGC 1052 and PKS 2254-367 being closer. Spectral variations of the radio continuum led Tingay et al. (2015) to argue for variable free-free absorbing (FFA) and ionized foreground material as opposed to synchrotron-self absorption (SSA) of jet-intrinsic plasma. High-spatial-resolution Very Long Baseline Interferometry (VLBI) observations at $22 \mathrm{GHz}$ (Tingay \& de Kool 2003) and $8.4 \mathrm{GHz}$ (Ojha et al. 2004, 2010) consistently confirm a compact double structure of $\sim 10$ mas diameter, that is, $\sim 3 \mathrm{pc}$ at a luminosity distance of $64.3 \mathrm{Mpc}(z \sim 0.014428 \pm 0.000023$; Meyer et al. 2004). This compact radio morphology with two distinct hot spots classifies PKS 1718-649 as a compact symmetric object (CSO). Tingay et al. (2002) provide an upper limit on the separation speed of $\lesssim 0.08 \mathrm{c}$. Together with the compact extent of the radio structure, this translates to an approximate age of $\gtrsim 60 \mathrm{yr}$. At an age of only hundreds to thousands of years, CSOs, where small advance speeds of terminal hotspots have been constrained (Owsianik \& Conway 1998), are therefore considered to be young. An extended jet (similar to NGC 1052) beyond the known parsecscale VLBI structure of PKS 1718-649 seems unlikely. The ATCA data presented by Maccagni et al. (2014) are also consistent with a point source. Given the $u v$-coverage of the Murchison Widefield Array (MWA), we can spectrally exclude extended radio emission at hundreds of megahertz down to arcsecond scales. In the image plane, however, we are not sensitive to faint remnant radio structures on these scales - a regime that the future 
Table 1. List of the X-ray observations used in this Letter.

\begin{tabular}{llllcc}
\hline \hline Abbrv. Date & obsid & det & exp [ks] & cnts $\left[\times 10^{2}\right]$ \\
\hline Ch 1 & $2010-11-09$ & 12849 & ACIS-S & 4.8 & 3.1 \\
Ch 2 & $2014-06-20$ & 16070 & ACIS-S & 15.9 & 14.2 \\
Ch 3 & $2014-06-23$ & 16623 & ACIS-S & 33.0 & 35.3 \\
XMM & $2017-03-05$ & 0784530201 & EPIC-pn & 20.3 & 50.7 \\
\hline
\end{tabular}

observatories MeerKAT and SKA are able to probe. Moreover, PKS 1718-649 is the first young radio galaxy confirmed to be $\gamma$-ray bright (Migliori et al. 2016).

The inverted radio spectrum below a few gigahertz and the morphology of PKS 1718-649 shape the picture of an active galactic nucleus (AGN) embedded in a cocoon of ionized matter. Recently, Maccagni et al. $(2014,2016,2018)$ also provided evidence for circumnuclear and clumpy molecular matter that is feeding the new-born AGN. While the line of sight is piercing this obscuring matter, both an inverted radio and photo-absorbed $\mathrm{X}$-ray continuum are expected. Studying the X-ray emission alongside with the radio emission is therefore a valuable tool for a better understanding of the environment of this young AGN (see also Müller et al. 2016, 2015 for a radio and X-ray study of the other $\gamma$-ray loud young radio galaxy PMN J1603-4904). While we also provide measures for the X-ray continuum absorption, here we primarily concentrate on the X-ray emission detected from the environment of PKS 1718-649.

An exemplary CSO, where extended X-ray emission could be investigated with the unprecedented spatial resolution of Chandra in great detail, is NGC 1052 (Kadler et al. 2004; Boeck 2012). Here, a double-sided radio jet reaches into the kiloparsec (kpc)-scale environment and is observed to align with collisionally ionized X-ray-bright gas. Siemiginowska et al. (2016) were the first to systematically study the X-ray signatures in a large number of CSOs. Within their sample, extended kiloparsec-scale X-ray emission could only be detected for the nearby source PKS 1718-649. To date, the lack of sufficient count statistics, however, has made it impossible to unveil the nature of this X-ray emitting gas. In this Letter, we present novel results from a recent XMM-Newton observation and a stack of three archival Chandra datasets, which combine the large effective area of XMM-Newton with the imaging capabilities of Chandra.

We use the cosmological parameters $\Omega_{\mathrm{m}}=0.308$, $\Omega_{\lambda}=0.692$, and $H_{0}=67.8 \mathrm{~km} \mathrm{~s}^{-1} \mathrm{Mpc}^{-1}$ (Planck Collaboration XIII 2016) and find a correspondence of $1 \operatorname{arcsec} \sim 312 \mathrm{pc}$.

\section{Observations and data reduction}

We base our analysis on four archival X-ray observations (three by Chandra and one by XMM-Newton, all listed in Table 1).

For all Chandra observations, the source is observed with the back-illuminated chip S3 of the ACIS-S CCD (Garmire et al. 2003). The VFAINT mode was used to most effectively screen cosmic ray events. We make use of CIAO v.4.8.1 and CALDB v.4.7.2 to reprocess event files with the task chandra_repro and extract spectra using specextract from within regions of $14^{\prime \prime}$ radius around the source pointing center. We extract the background spectra from annuli of 30-44" radius. Pileup can be neglected after fitting an absorbed power-law based on the pileup-kernel in ISIS v.1.6.2-40. Data are only extracted between $0.5 \mathrm{keV}$ and $8 \mathrm{keV}$ with maximal effective area. We rebin each Chandra spectrum to 4,6 , and 8 channels per bin within $0.5-1 \mathrm{keV}, 3-5 \mathrm{keV}$, and $5-8 \mathrm{keV}$, respectively. That way the grid oversamples the spectral resolution by no more than a factor of three (Kaastra \& Bleeker 2016). We exclude a point source at RA: $17^{\mathrm{h}} 23^{\mathrm{m}} 42^{\mathrm{s}}$, Dec: $-65^{\circ} 00^{\prime \prime} 23^{\prime}$ and extract surface brightness profiles using dmextract with 15 annuli of $1^{\prime \prime}$ width and $0.5+n^{\prime \prime}$ radius $(n=0 \ldots 14)$, each centered at the source center. The background from an annulus between $30^{\prime \prime}$ and $60^{\prime \prime}$ is subtracted from the profile. The Chandra PSF is simulated for the spectrum Ch 2 by combining 25 runs of ChaRT. For each realization, the PSF is projected onto the detector plane using MARX. The resulting angular resolution is limited by the detector pixel size of $\sim 0.5^{\prime \prime}(\sim 156 \mathrm{pc})$ at the given distance of PKS 1718-649.

We observed PKS 1718-649 using XMM-Newton/EPIC-pn (Villa et al. 1996; Meidinger et al. 1996; Strüder et al. 2001) in Large Window mode and extracted the count spectrum using SAS v.16.0.0. After creating calibrated event lists with filtered hot and bad pixels, events in the range $7-15 \mathrm{keV}$ are screened for particle flaring with a threshold of $8 \mathrm{cnts} \mathrm{ks}^{-1} \mathrm{arcmin}^{-2}$. We extract source counts from a circular region of $40^{\prime \prime}$ radius for EPIC-pn and background counts from an off-source region of $49^{\prime \prime}$ radius. The task epatplot returns no signs for pileup and we consider all counts between 0.3 and $10 \mathrm{keV}$. Following the same strategy as for Chandra, we apply a geometrical binning with factors of 5, 6, 10 and 20 in the ranges $0.3-1.5 \mathrm{keV}$, $1.5-2 \mathrm{keV}, 2-7 \mathrm{keV}$, and $7-10 \mathrm{keV}$, respectively.

\section{X-Ray image and spectral analysis}

In order to quantify the extended and non-variable X-ray emission that has been detected by Siemiginowska et al. (2016) based on the Chandra observation $\mathrm{Ch} 1$, we study a stacked image consisting of $\mathrm{Ch} 1$ and the more recent observations $\mathrm{Ch} 2$ and $\mathrm{Ch} 3$. The stacking was performed using the standard CIAO task merge_obs. Figure 1 (left and right panels) shows that the bulk of the photons and in particular hard X-rays above $\sim 1.5 \mathrm{keV}$ are emitted from the unresolved core region with an excess of $\sim 60 \%$ above the soft X-rays within a radius of $3^{\prime \prime}$. The PSF is encircling $99 \%$ of the point-source flux within $\sim 3^{\prime \prime}$. In contrast, soft $(\sim 0.3-1.5 \mathrm{keV}) \mathrm{X}$-ray emission exceeds that in the hard band by $\sim 54 \%$ in the extended region between $\sim 3$ and $\sim 8^{\prime \prime}$.

Despite the unprecedented spatial resolution of Chandra, the soft-X-ray effective area of XMM-Newton makes up twice and ten-times the area of Chandra/ACIS at around $1 \mathrm{keV}$ and $0.5 \mathrm{keV}$, respectively. We therefore fit the Chandra spectra combined with recently acquired $X M M-N e w t o n$ data to unveil the origin of the extended emission (see Fig. 2). The hard-X-ray data follow a common power-law of constant photon index $(\Gamma=1.78)$ that is absorbed towards lower energies. Given the limited number of counts, we are not sensitive to the continuum signatures of a possible ionized absorber and instead apply a model for neutral absorbing gas (tbabs). Above this continuum, XMM-Newton allows us to confirm an emission feature consistent with $\mathrm{O}$ VII around $0.56-0.57 \mathrm{keV}$ and a broad emission complex around $0.7-0.9 \mathrm{keV}$, likely due to $\mathrm{Ne}$ and $\mathrm{Fe}$ $(\sim 0.5-0.6 \mathrm{keV}$ and $\sim 0.6-1.2 \mathrm{keV})$. The former is described with the photoionized plasma component xstar (Bautista \& Kallman 2000; Porquet \& Dubau 2000; Bautista \& Kallman 2001) for a nuclear irradiating power-law as measured, and an ambient gas density of $10^{4} \mathrm{~cm}^{-3}$ (Tingay et al. 2015). We find the ionization parameter to be $\log \xi=0.04_{-0.05}^{+1.13}$. The latter broad feature is best described with emission of a collisionally ionized plasma $\left(k T=0.75_{-0.08}^{+0.07} \mathrm{keV}\right)$ using apec (Smith et al. 2001). This component dominates the soft X-rays and must therefore account for 

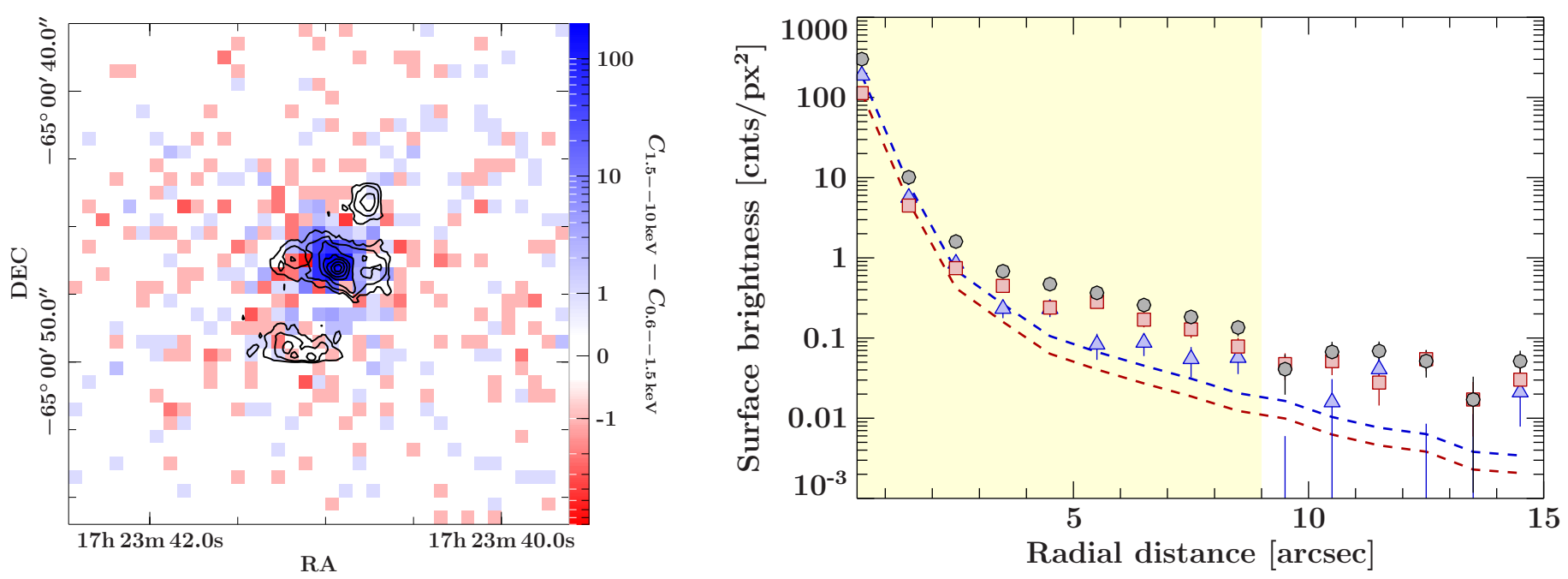

Fig. 1. Left: difference map between the hard (1.5-10 keV) and soft $(0.3-1.5 \mathrm{keV})$ band including counts of Ch 1 , Ch 2 , and Ch 3 . Positive pixels (blue) depict a hard excess, negative pixels (red) a soft excess. Overlayed contours in black show $\mathrm{H}_{2} 1-0 \mathrm{~S}(1)$ emission (Maccagni et al. 2016). Right: surface brightness profiles for the stacked map for the energy intervals $0.5-10 \mathrm{keV}$ (black/circles), $0.3-1.5 \mathrm{keV}$ (red/squares) and $1.5-10 \mathrm{keV}$ (blue/triangles). The blue and red dashed lines show the Chandra PSF normalized to the hard (1.5-10 keV) and soft (0.3-1.5 keV) X-ray profile. The yellow shaded section marks the extent of the map on the left.

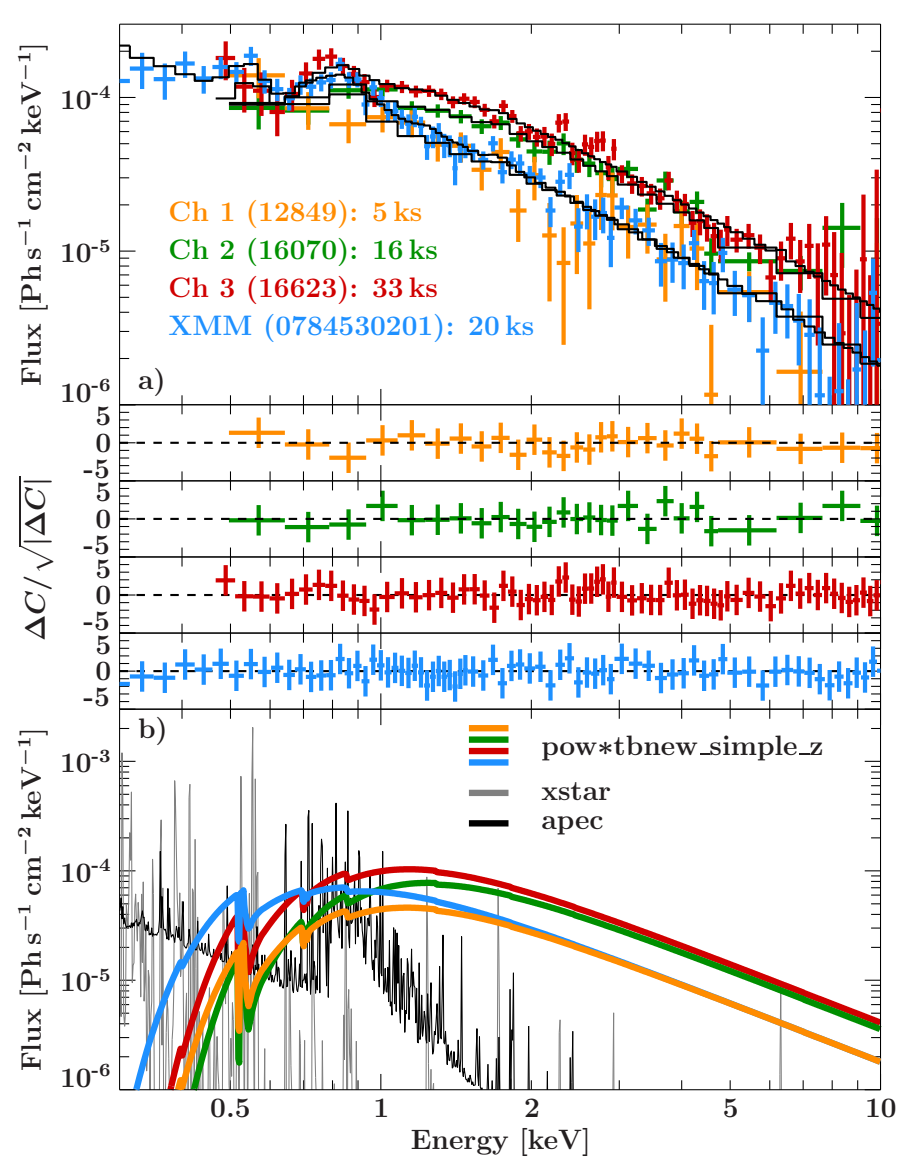

Fig. 2. Panel $a$ : unfolded spectra for the observations Ch 1,2, 3 and $\mathrm{XMM}$ in color with the individual model evaluations in black on top. The residuals are shown below. Panel b: model evaluations of the variable absorbed power law in color and the non-variable emission plasmas in gray (xstar) and black (apec).

a significant portion of the extended emission in Fig. 1. The low source flux prevents a detailed line-diagnostic study with XMM-Newton/RGS.
Table 2. Best-fit parameters for the simultaneous fit of the data from Table 1.

\begin{tabular}{lllll}
\hline \hline Abbrv. & $\begin{array}{l}\text { pow } \\
\text { norm }\end{array}$ & $\Gamma$ & $\begin{array}{l}\text { tbabs } \\
N_{\mathrm{H}}\left[\times 10^{22} \mathrm{~cm}^{-2}\right]\end{array}$ & $\begin{array}{l}\text { Flux }(0.5-10 \mathrm{keV}) \\
{\left[\times 10^{-13} \mathrm{erg} \mathrm{cm}^{-2} \mathrm{~s}^{-1}\right]}\end{array}$ \\
\hline Ch 1 & $\left(1.1_{-0.2}^{+0.3}\right) \times 10^{-4}$ & $1.78_{-0.09}^{+0.10}$ & $0.5_{-0.2}^{+0.3}$ & 5.4 \\
Ch 2 & $(2.2 \pm 0.3) \times 10^{-4}$ & $“$ & $0.69_{-0.14}^{+0.18}$ & 9.9 \\
Ch 3 & $(2.5 \pm 0.3) \times 10^{-4}$ & “ & $0.55_{-0.11}^{+0.14}$ & 11.6 \\
XMM & $\left(1.09_{-0.11}^{+0.15}\right) \times 10^{-4}$ & “ & $0.31_{-0.09}^{+0.14}$ & 5.8 \\
\hline
\end{tabular}

Notes. All spectra are fitted with apec (norm $2.0_{-0.5}^{+0.6} \times 10^{-5}$; $\left.k T=0.75_{-0.08}^{+0.07} \mathrm{keV}\right)$ and xstar $\left(N_{\mathrm{H}}=1 \times 10^{25} \mathrm{~cm}^{-2}\right.$ based on an ambient gas density of $10^{4} \mathrm{~cm}^{-3}$ and an assumed path length of $10 \mathrm{pc}$; see Tingay et al. (2015); $\left.\log \xi=0.04_{-0.05}^{+1.13}\right)$. The unit of the apec normalization is $\left[10^{-14} / 4 \pi\left(D_{\mathrm{A}}(1+z)\right)^{2}\right] \int n_{\mathrm{e}} n_{\mathrm{H}} \mathrm{d} V$ (see the apec manual for details). Overall, we apply a redshift of $z=0.0144$ (Doyle et al. 2005) and a Galactic column density of $5.7 \times 10^{19} \mathrm{~cm}^{-2}$ (Kalberla et al. 2005).

Besides the non-variable emission components xstar and apec, the only parameters that turn out to be variable (on time scales of years) are the source-intrinsic column density $\left(N_{\mathrm{H}}=0.3-0.7 \times 10^{22} \mathrm{~cm}^{-2}\right)$ and flux of the incident hard X-ray power law $\left(5.4-11.6 \times 10^{-13} \mathrm{erg} \mathrm{cm}^{-2} \mathrm{~s}^{-1}\right)$. We use Cash statistics due to the low number of counts in Ch 1 and $\mathrm{Ch} 2$. The simultaneous fit describes all four data sets well with $\mathrm{C}(\mathrm{dof})=444(439)$. The resulting parameters and uncertainties are listed in Table 2.

\section{Discussion}

We are able to report, for the first time in the literature, on variable X-ray absorption and on the physics of the extended X-ray emission in the CSO PKS 1718-649 that is for the most part unrelated to nuclear feedback. We stacked all archival Chandra data available for this object, and analyzed the combined spectra of Chandra and XMM-Newton. Our results form a two-fold picture. The bulk of the unresolved core emission comprises Comptonized hard X-rays. The X-ray source may be a corona close to the accretion disk (e.g., Dove et al. 1997, and references therein), the parsec-scale radio jet, or its jet base 
(e.g., Markoff et al. 2005; Dauser et al. 2013). The spatially resolved and extended environment beyond $\sim 600 \mathrm{pc}$ from the core, however, predominantly emits soft X-rays.

Our proposed XMM-Newton observation provides a sufficient amount of counts below $1 \mathrm{keV}$ to investigate the nature of this extended and soft emission. We detect two plasma emission components that are non-variable on the probed time-scales of years: One is photoionized by the incident continuum up to a level of $\log \xi=0.04_{-0.05}^{+1.13}$, and the second dominates the soft emission and is best described as a collisionally ionized and hot gas $\left(k T=0.75_{-0.08}^{+0.07} \mathrm{keV}\right.$ or $\left.T=(7.8-9.5) \times 10^{6} \mathrm{~K}\right)$. A significant contribution of reflected photons off the accretion disk or surrounding matter can be tentatively ruled out: related codes (e.g., xillver, García et al. 2013) predict strong signatures of $\mathrm{Fe} \mathrm{K} \alpha$ at $\sim 6.4 \mathrm{keV}$ as well as $\mathrm{Mg}$, $\mathrm{Si}$, and $\mathrm{S}$, which remains in contrast to our observations of highly ionized $\mathrm{O}$ and $\mathrm{Ne} / \mathrm{Fe}$ at $\lesssim 1 \mathrm{keV}$. Additional contributions to the soft X-rays can likely be neglected. First, scattered radiation off the extended gaseous and dusty environment is not required in our fits. Second, the luminosity of a population of X-ray binaries would lie roughly two orders of magnitude below the measured $0.5-8 \mathrm{keV}$ luminosity given the weak star formation rate (SFR) of PKS 1718-649 (Willett et al. 2010; Mineo et al. 2012).

We now discuss the origin of these two components of gas. In the optical, Filippenko (1985) cross-identified the lowluminosity AGN PKS 1718-649 with a low-ionization nuclear emission-line region (LINER). In particular, the detection of [O III] brings up the question of whether this emission could originate co-spatial and in pressure equilibrium with the photo-ionized gas component that we model with xstar in PKS 1718-649 (e.g., Bianchi et al. 2006; Chakravorty et al. 2009). Filippenko (1985) measure an [O III] intensity ratio of $(\lambda 4959+\lambda 5007) / \lambda 4363 \sim 12$, which implies temperatures of $\gtrsim 6.2 \times 10^{4} \mathrm{~K}$ (Proxauf et al. 2014). At these temperatures, collisional ionization is the dominant process and likely related to the parsec-scale jet. In the absence of jet-shocked regions, however, nuclear irradiation is still influential. Our xstar modeling implies a highly photoionized plasma on sub-parsec scales, traced by $\mathrm{O}$ VII. At larger distances, the nuclear ionizing potential will decrease and lead to a stratification of differently ionized species of oxygen. While we are lacking spatial information on the distribution of [O III] in PKS 1718-649, Ricci et al. (2015) find [O III] to be distributed at around $100 \mathrm{pc}$ in a sample of LINER galaxies, consistent with the size of a still expanding Strömgen sphere in GPS sources (Vink et al. 2006). This photoionized environment shifts to kiloparsecs in evolved AGN (e.g., Rodríguez-Ardila et al. 2006).

In PKS 1718-649, we spatially resolve a collisionally ionized gas phase at $\lesssim \mathrm{kpc}$ distances. Based on its low overall luminosity with $(2.5-5.4) \times 10^{41} \mathrm{erg} \mathrm{s}^{-1}$, we conclude that this gas must be part of the extended and hot interstellar medium, spatially distinct from the photoionized gas phase traced by $\mathrm{O}$ VII. In the CSO NGC 1052, the soft X-ray emission on kiloparsec scales is well explained by jet excitation (Kadler et al. 2004; Boeck 2012). This is most likely negligible in PKS 1718-649, where the parsec-scale radio jet is too compact to drive sufficiently energetic shocks into the interstellar medium (ISM) and to heat up this gas. This is in line with the non-detection of coronal lines (Filippenko 1985; Rodríguez-Ardila et al. 2006) and the lack of notable asymmetries of the X-ray emission on such large scales. We therefore propose an alternative scenario, where supernovae $(\mathrm{SNe})$ in the host galaxy can feed the galactic halo with hot and X-ray-bright gas (see also, e.g., Strickland et al. 2004, for similar conclusions based on a sample of star forming disk galaxies).
Supernovae in the host galaxy are supported by the observation of active star formation in PKS 1718-649 via $\mathrm{H} \alpha$ and PAH (Polycyclic Aromatic Hydrocarbon) emission (Kennicutt 1983; Maccagni et al. 2014; Willett et al. 2010). Sullivan et al. (2006) study star-forming host galaxies of 100 confirmed SNe Ia. Their results imply a SN rate of $\sim 3.2 \times 10^{-2}$ galaxy $^{-1} \mathrm{yr}^{-1}$ for a galactic stellar mass of $M_{*} \sim$ $4.9 \times 10^{11} M_{\odot}$ for PKS 1718-649 (Maccagni et al. 2014). When considering a SFR of $\sim 0.8-1.9 M_{\odot} \mathrm{yr}^{-1}$ (Willett et al. 2010, using PAH signatures ${ }^{1}$ ), Sullivan et al. (2006) infer a SN rate of $\sim 0.7-1.3 \times 10^{-3}$ galaxy $^{-1} \mathrm{yr}^{-1}$. Very similar SN rates, namely $\left(4 \times 10^{-3}-4 \times 10^{-2}\right)$ galaxy $^{-1} \mathrm{yr}^{-1}$, can explain the diffuse X-ray emission of M 81 (Shelton 1998; Page et al. 2003), which is classified as LINER as well (Heckman 1980). Moreover, the gas temperature that Page et al. (2003) determine for M 81 corresponds well to that measured in our work.

An independent indicator for the presence of $\mathrm{SNe}$ is given by the emission of different forms of hydrogen. While $\mathrm{H}_{2}$ in the ISM is generally too cold to emit, Maccagni et al. (2016) observe a disk-like distribution in the inner few arcseconds $(\lesssim \mathrm{kpc})$ of PKS 1718-649. Roughly perpendicular to it, an outer disk of $\mathrm{H}_{2}$ at distances larger than $650 \mathrm{pc}$ aligns with neutral $\mathrm{H} \mathrm{I}$ (Maccagni et al. 2014) and $\mathrm{H} \alpha$ (Keel \& Windhorst 1991). Among the several $\mathrm{H}_{2}$ excitation mechanisms at play (e.g., Maloney et al. 1996; Rodríguez-Ardila et al. 2004; Dors et al. 2012, and references therein), Maccagni et al. (2018) favor nuclear, non-thermal $\mathrm{X}$-rays for the inner few parsecs. Besides that, shock excitation by the parsec-scale jet or nuclear UV radiation may also play a role in this compact environment. At larger distances of hundreds to thousands of parsecs, $\mathrm{H}_{2}$ appears co-spatial with and most likely excited by the warm and diffuse soft X-ray-emitting gas (Fig. 1), which we suggest to be due to the direct influence of SNe. The excitation can, however, to some smaller degree also arise due to nuclear UV/X-ray emission or UV photons of dense molecular star-forming regions (Puxley et al. 1990; Davies et al. 1998).

\section{Conclusions}

In this Letter, we investigated the nature of the extended X-ray emitting gas in PKS 1718-649. By stacking all archival Chandra data, we find this gas to primarily emit in soft X-rays. Our recent observation by $X M M-N e w t o n$ and its large effective area allow us to perform a detailed spectral analysis of this emission. Besides a photoionized $\left(\log \xi=0.04_{-0.05}^{+1.13}\right)$ gas phase on subparsec scales, the bulk of the soft X-rays is emitted by diffuse, hot $\left(T=(7.8-9.5) \times 10^{6} \mathrm{~K}\right)$, and collisionally ionized gas that dominates the nuclear emission in the range $\sim 1-2.8 \mathrm{kpc}$. We argue that supernovae are plausible candidates to power this region as opposed to the overly compact parsec-scale jets of the young AGN. This conclusion is driven from observations of active star formation in PKS 1718-649, estimates on the expected SN rate, as well as the theoretically predicted X-ray flux of SN remnants.

Acknowledgements. We thank the internal referee at MPIfR, S. Komossa, for her comments that helped to significantly improve the clarity of this Letter. We are also grateful for fruitful discussions with S. Toonen (on stellar evolution and supernova progenitors), M. Sasaki, P. Zhou, and J. Vink (on supernova remnants), as well as N. Przybilla (on atomic data and [O III] diagnostics). T.B. acknowledges support by the DLR grant DLR-50-OR-1606 and by the Dutch Research School for Astronomy (NOVA). A.R.A thanks to CNPq for partial support to this work. F.K. acknowledges funding from the European Union's Horizon 2020 research and innovation program under grant agreement No. 653477. R.S. and F.P. gratefully acknowledge support from the

$1 \mathrm{PAH}$ features at $6.2 \mu \mathrm{m} \quad(E W=0.04 \mu \mathrm{m})$ and $11.3 \mu \mathrm{m}$ $(E W=0.13 \mu \mathrm{m})$ are measured with Spitzer/IRS and a slit of $11^{\prime \prime} \times 168^{\prime \prime}$. 
European Research Council under the European Union's Seventh Framework Programme (FP/2007-2013)/ERC Advanced Grant RADIOLIFE-320745. A.K. and M.L. acknowledge the support of the Bundesministerium für Wirtschaft und Technologie (BMWi) through Deutsches Zentrum für Luft- und Raumfahrt (DLR) grant 50OR1709. E.R. was partially supported by the Spanish MINECO grant AYA2015-63939-C2-2-P and by the Generalitat Valenciana grant PROMETEOII/2014/057. We thank J. E. Davis for the development of the slxfig module that has been used to prepare the figures in this work We made use of ISIS functions provided by ECAP/Remeis observatory and MIT (http://www.sternwarte.uni-erlangen.de/isis/) as well as the NASA/IPAC Extragalactic Database (NED), which is operated by the Jet Propulsion Laboratory, California Institute of Technology, under contract with the National Aeronautics and Space Administration.

\section{References}

Bautista, M. A., \& Kallman, T. R. 2000, ApJ, 544, 581

Bautista, M. A., \& Kallman, T. R. 2001, ApJS 134, 139

Bianchi, S., Guainazzi, M., Chiaberge, M. 2006, A\&A 448, 499

Boeck, M. 2012, Ph.D. Thesis, Universität Erlangen-Nürnberg

Chakravorty, S., Kembhavi, A. K., Elvis, M., et al. 2009, MNRAS 393, 83

Dauser, T., Garcia, J., Wilms, J., et al. 2013, MNRAS 430, 1694

Davies, R. I., Sugai, H., \& Ward, M. J. 1998, MNRAS 300, 388

Dors, Jr. O. L., Riffel, R. A., Cardaci, M. V., et al. 2012, MNRAS 422, 252

Dove, J.B., Wilms, J., Maisack, M., \& Begelman, M. C. 1997, ApJ, 487, 759

Doyle, M. T., Drinkwater, M. J., Rohde, D. J., et al. 2005, MNRAS, 361, 34

Filippenko, A. V. 1985, ApJ 289, 475

García, J., Dauser, T., Reynolds, C. S., et al. 2013, ApJ, 768, 146

Garmire, G. P., Bautz, M. W., Ford, P. G., et al. 2003, in X-Ray and GammaRay Telescopes and Instruments for Astronomy, eds. J. E. Truemper, \& H. D Tananbaum, Proc. SPIE, 4851, 28

Heckman, T. M. 1980, A\&A 87, 152

Jauncey D. L., Tingay, S. J., Preston, R. A., et al. 1998, in Radio Emission from Galactic and Extragalactic Compact Sources, IAU Colloq., 164 eds. J. A. Zensus, G. B. Taylor, \& J. M. Wrobel, ASP Conf. Ser., 144, 193

Kaastra, J. S., \& Bleeker, J. A. M. 2016, A\&A 587, A151

Kadler, M., Kerp, J., Ros, E., et al. 2004, A\&A 420, 467

Kalberla, P. M. W., Burton, W. B., Hartmann, D., et al. 2005, A\&A, 440, 775

Keel, W. C., \& Windhorst, R. A. 1991, ApJ, 383, 135

Kennicutt, Jr. R. C. 1983, ApJ, 272, 54

Maccagni, F. M., Morganti, R., Oosterloo, T. A., Mahony, E. K. 2014, A\&A 571,
Maccagni, F. M., Santoro, F., Morganti, R., et al. 2016, A\&A 588, A46

Maccagni, F. M., Morganti, R., Oosterloo, T. A., et al. 2018, A\&A, in press, DOI: 10.1051/0004-6361/201732269

Maloney, P. R., Hollenbach, D. J., Tielens, A. G. G. M. 1996, ApJ, 466, 561

Markoff, S., Nowak, M. A., Wilms J. 2005, ApJ, 635, 1203

Meidinger, N., Braeuninger, H. W., Hartmann, R., et al. 1996, in EUV, X-Ray, and Gamma-Ray Instrumentation for Astronomy VII, eds. O. H. Siegmund, \& M. A. Gummin, Proc. SPIE, 2808, 492

Meyer, M. J., Zwaan, M. A., Webster, R. L., et al. 2004, MNRAS 350, 1195

Migliori, G., Siemiginowska, A., Sobolewska, M., et al. 2016, ApJ, 821, L31

Mineo, S., Gilfanov, M., Sunyaev, R. 2012, MNRAS 419, 2095

Müller, C., Burd, P.R., Schulz, R., et al. 2016, A\&A 593, L19

Müller, C., Krauß, F., Dauser, T., et al. 2015, A\&A 574, A117

Ojha, R., Fey, A. L., Johnston, K. J., et al. 2004, AJ, 127, 3609

Ojha, R., Kadler, M., Böck, M., et al. 2010, A\&A 519, A45

Owsianik, I., \& Conway, J. E. 1998, A\&A 337, 69

Page, M. J., Breeveld, A. A., Soria, R., et al. 2003, A\&A 400, 145

Planck Collaboration XIII. 2016, A\&A 594, A13

Porquet, D., Dubau, J. 2000, A\&AS, 143, 495

Proxauf, B., Öttl, S., Kimeswenger, S. 2014, A\&A 561, A10

Puxley, P. J., Hawarden, T. G., Mountain, C. M. 1990, ApJ, 364, 77

Ricci, T. V., Steiner, J. E., Menezes, R. B. 2015, MNRAS 451, 3728

Rodríguez-Ardila, A., Pastoriza, M. G., Viegas, S., et al. 2004, A\&A 425, 457

Rodríguez-Ardila, A., Prieto, M. A., Viegas, S., \& Gruenwald, R. 2006, ApJ, 653, 1098

Shelton, R. L. 1998, ApJ, 504, 785

Siemiginowska, A., Sobolewska, M., Migliori, G., et al. 2016, ApJ, 823, 57

Smith, R. K., Brickhouse, N. S., Liedahl, D. A., \& Raymond J. C. 2001, ApJ, 556, L91

Strickland, D. K., Heckman, T. M., Colbert, E. J. M., et al. 2004, ApJS 151, 193

Strüder, L., Briel, U., Dennerl, K., et al. 2001, A\&A 365, L18

Sullivan, M., Le Borgne, D., Pritchet, C. J., et al. 2006, ApJ, 648, 868

Tingay, S. J., \& de Kool, M. 2003, AJ, 126, 723

Tingay, S. J., Jauncey, D. L., Reynolds, J. E., et al. 1997, AJ, 113, 2025

Tingay, S. J., Reynolds, J. E., Tzioumis, A. K., et al. 2002, ApJS 141, 311

Tingay, S. J., Macquart, J. P., Collier, J. D., et al. 2015, AJ, 149, 74

Villa, G. E., Abbey, A. F., Arnaud, M., et al. 1996, in EUV, X-Ray, and GammaRay Instrumentation for Astronomy VII, eds. O. H. Siegmund, \& M. A. Gummin, Proc. SPIE, 2808, 402

Vink, J., Snellen, I., Mack, K. H., \& Schilizzi, R. 2006, MNRAS 367, 928

Willett, K. W., Stocke, J. T., Darling, J., Perlman, E. S. 2010, ApJ, 713, 1393 